\title{
Die christologische Forschung in der gegenwärtigen nordamerikanischen Theologie
}

Prof. Dr. Hans Schwarz, Rilkestraße 44, 8411 Lappersdorf

Christologie ist Lehre von Christus. Aber als Lehre kann sie nicht unter Absehen von Lehrenden und Lernenden betrieben werden, soll nicht ihr Bezug zur Wirklichkeit verloren gehen. Deshalb ist Christologie, wie Theologie insgesamt, durch die sozialkulturelle Situation des Menschen bestimmt. So hat etwa Kosuke Koyama eine typisch asiatische Water Buffalo Theology verfaßt ${ }^{1}$ und der Standing Rock Sioux Indianer Vine Deloria Jr. machte uns darauf aufmerksam, daß Gott rot sei. ${ }^{2}$ In ähnlicher Weise müssen wir die Befreiungstheologien von Gustavo Gutiérrez, Hugo Assmann oder Denis Goulet verstehen. Auch die nordamerikanische christologische Forschung bringt in zweifacher Hinsicht ein bestimmtes situationsgebundenes Kolorit mit sich: Zunächst spiegelt die christologische Ausdrucksweise die sozialen und ethnischen Spannungen im eigenen Land wider, wie etwa die schwarze Theologie oder die Befreiungstheologie der Frauen. Zum anderen ist Amerika durch einen denominationalen, theologischen und religiösen Pluralismus gekennzeichnet, der sich in einer Spannweite äußert, die von einer radikalen Inkarnationstheologie bis zur literalistischen und spekulativen Eschatologie $\mathrm{Hal}$ Lindseys reicht. Als Eigenleistung besonderer Art muß noch die Prozeßchristologie genannt werden, die jetzt auch in Deutschland Interesse erweckt.

\section{Die christologischen Strömungen der Gegenwart}

Beeindruckend in der gegenwärtigen christologischen Diskussion, aber keineswegs unerwartet für Kenner Nordamerikas, ist die Bedeutung der Soteriologie. Wenn Jesus oder Christus für etwas steht, dann drückt sich in ihm Heil, Erlösung oder auch Befreiung aus. Das wird nirgendwo so deutlich, wie in den sozial und ethnisch geprägten Christologien.

\section{a) Von der schwarzen Theologie zur Befreiungstheologie der Frauen}

Wir wollen hier nicht auf Details der Befreiungstheologie eingehen. Die Literatur darüber ist inzwischen so angewachsen, daß mancherorts sogar schon Lehrstühle für Liberation Theology eingerichtet wurden. James H. Cone beginnt sein Buch Black Theology and Black Power (dt.: Schwarze Theologie) mit der Feststellung: "Die Tatsache, daß ich schwarz

\footnotetext{
Kosuke Koyama, Water Buffalo Theology, Maryknoll, New York 1974.

2 Vine Deloria, Jr., God is Red, New York 1973.
} 
bin, (ist) meine letztgültige Wirklichkeit (ultimate reality)«. ${ }^{3}$ Als Schwarzer behauptet dann Cone, daß eine höhere letztgültige Wirklichkeit für Schwarze nur sinnvoll sein kann, wenn sie zu dem innersten Kern des Schwarzseins in Beziehung steht. Obwohl das Christentum zu den Schwarzen durch die weißen Herren kam, die verlangten, daß die Schwarzen ihr Interesse an dieser Welt und auch an der Schwarzheit aufgeben und statt dessen das Jenseits und das Weißsein betonen, beginnt nach Cone das Christentum mit dem Menschen Jesus und gipfelt in seinem Leben, Tod und Auferstehung. In Jesus begibt sich Gott in die Tiefe der menschlichen Existenz mit der alleinigen Absicht, die Ketten der Sklaverei abzustreifen und den Menschen von den widergöttlichen Herrschaften und Mächten zu befreien, die sein Verhältnis zu Gott belasten. "Jesu Werk ist ein Werk der Befreiung ". Indem Jesus zum Sklaven wird, öffnete er die Wirklichkeit der menschlichen Existenz, die uns vorher verschlossen war. Durch die Begegnung mit Jesus erkennen wir die volle Bedeutung des Wirkens Gottes in der Geschichte und den Ort des Menschen in ihr. Da Gott sich in Christus auf die Seite der Unterdrückten stellt, werden die Armen dazu befreit, sich gegen das aufzulehnen, was sie in ihrer Menschlichkeit beschneidet. Durch die Botschaft Jesu wird das Christentum eine Religion des Protests gegen das Leiden und die Unterdrückung des Menschen. Christus, sagt Cone, kann nicht in das erste Jahrhundert verbannt werden, denn er wird als Zeitgenosse erkannt, der den Gefangenen Befreiung verkündet und gegen alle die protestiert, die schweigend die Strukturen der Ungerechtigkeit hinnehmen. Das Evangelium bezeugt einen Gott, der jetzt bei uns ist und tatkräftig die Mächte bekämpft, die den Menschen gefangennehmen wollen. Cone erkennt: "Obwohl die entscheidende Schlacht gegen das Böse geschlagen und gewonnen wurde, ist der Krieg jedoch noch nicht zu Ende. " Der Kampf zwischen Christus und Satan geht weiter, denn die Menschen sind von den Mächten des Bösen geknechtet, die sie zu Sklaven machen wollen.

In Christus frei zu sein bedeutet, daß der Mensch vom Joch des Gesetzes als des angeblichen Garanten des Heils befreit und in ein freies erwachsenes Lebensverhältnis mit Gott und dem Nächsten gestellt wird. Dies ist das Ziel des Menschseins, wie es uns Christus gezeigt hat. Doch in dieser Welt, die in ständigem Konflikt mit Christus lebt, bringt das auch Leiden mit sich und keineswegs ein Schlaraffenland.

${ }^{3}$ James H. Cone, Schwarze Theologie. Eine christliche Interpretation der Black-PowerBewegung, mit einem Nachw. v. Frederick Herzog, u. ausgew. Dokumenten, übers. v. Ulrich G. Fick, München 1971, S.43. Vgl. auch ders. A Black Theology of Liberation, Philadelphia 1970, S. 197-227, u. "Who Is Jesus Christ for Us Today?", Christianity and Crisis, Bd. XXXV (Apr. 14, 1975), 81-85.

4 James H. Cone, Schwarze Theologie, S. 45.

5 Ebd., S. 50. 
Um durch Christus gerecht zu werden, wird man wie er in Situationen gestellt, wo man wie Christus für die Armen, für Gott und gegen die Welt sein muß. Wenn man als Nichts vor Gott steht und durch Gnade in sein Reich angenommen wird, erkennt man als Christ, daß man gerecht gemacht wurde, um an Gottes Seite in der Schlacht für die Gerechtigkeit zu kämpfen. Doch darf die Gerechtigkeit nicht von der Liebe Gottes getrennt werden, denn Liebe verhütet, daß die Gerechtigkeit gesetzlich wird und Gerechtigkeit bewahrt die Liebe vor Sentimentalität. Liebe bedeutet, daß Gott die Übel der Menschheit ausbügelt, denn sie sind unvereinbar mit dem Ziel des Menschen, und Gerechtigkeit bedeutet, daß Gott sich nicht vom Übel abwendet, denn er gibt nicht vor, daß Unrecht Recht ist. So wirkt Gott durch Liebe und Gerechtigkeit zum Wohl des Menschen. Daß Gott den schwarzen Menschen liebt, heißt, daß der Schwarze sich nicht zu hassen braucht, weil er nicht weiß ist und auch nicht weiß werden muß. Wenn Paulus sagt, daß wir in Christus eine neue Kreatur sind, darf das ein erlöster schwarzer Mensch wörtlich nehmen, versichert uns Cone. ${ }^{6}$ Ein Schwarzer rühmt sich seiner Schwarzheit, nicht im egoistischen Sinne des Selbstruhms, sondern daß er sich als Schwarzer als Geschöpf Gottes erkennt. Auch die Nächstenliebe, besonders wenn sie den weißen Nächsten betrifft, ist davon berührt. Einen Weißen zu lieben heißt, ihm als Du zu begegnen, ohne als Schwarzer zu einem Es zu werden, obwohl der Weiße gewohnt ist, im Schwarzen ein Es zu sehen. Man darf deshalb die neugefundene Identität in Christus nicht aufgeben, denn echte Liebe kann es nur unter Gleichen geben. So gehören Liebe, Gerechtigkeit und Macht zusammen.

Wenn Cone und andere Vertreter der Schwarzen Theologie die Diesseitigkeit des Heils so nachdrücklich vertreten, ist es nicht verwunderlich, daß der eschatologische Aspekt des Heils im traditionellen Sinne zu kurz kommt. Nach Cone wurden die Schwarzen immer auf das Jenseits vertröstet. Doch »die Vorstellung des Himmels ist für die Schwarze Theologie ohne Bedeutung. Der Christ darf keine Zeit daran verschwenden, über die zukünftige Welt (falls es sie überhaupt gibt) zu kontemplieren «? Auch dürfen wir nicht unsere Augen vor der jetzigen Ungerechtigkeit abwenden, denn die Perlentore und die goldenen Straßen des Jenseits können uns nicht davon ablenken, daß die irdischen Straßen mit schwarzem Blut bedeckt sind. Schwarze Theologie, behauptet Cone, weiß, daß »echter biblischer Glaube die Eschatologie mit der Geschichte in Beziehung setzt, das heißt, mit dem, was Gott für sein Volk getan hat, tut, und tun wird. Nur auf Grund dessen, was Gott getan hat und was er jetzt tut, können wir überhaupt sinnvoll von der Zukunft sprechen ". ${ }^{*}$ Cone hat in seinem

\footnotetext{
6 Vgl. a. a. O., S. 57-64.

Ebd. S. 136.

8 Ebd. S. 137.
} 
christologischen Entwurf einer schwarzen Theologie die eschatologische Heilserfüllung nicht verneint, auch wenn er wenig Sinn für die biblischen Bilder dieser Erfüllung zeigt und die Heilsgegenwart stark betont. Zu fragen bleibt natürlich, ob die Gegenwart des Heils sich angesichts der Sündhaftigkeit des Menschen so ungetrübt aussagen läßt, wie es Cone versucht. Cones Entwurf hat viele Nachfolger gefunden, wie etwa in Gayraud S. Wilmore, der von Christus als dem schwarzen Messias spricht.' Andere meinen dagegen, daß Cone noch zu provinziell ist und wollen das Schwarzsein »zu einem universalen Symbol der menschlichen Unterdrükkung " machen. ${ }^{10}$

Die Schwarze Theologie ist in den letzten Jahren durch die Befreiungstheologie oder besser Christologie der Frauen etwas in den Hintergrund gedrängt worden. Dabei geht es nicht primär um Gleichberechtigung, sondern um die Befreiung der Frau von ihrer Stellung als entwürdigtes und erniedrigtes Sexsymbol. Die katholische Laientheologin Rosemary Radford Ruether erklärt deshalb auch die Freudsche Psychoanalyse als eine weitgehend säkularisierte Form des herkömmlichen religiösen Sexismus. ${ }^{11}$

Wenn man den Sexismus analysiert, dann zeigt sich nach ihr, daß das einseitige Abhängigkeitsverhältnis der Frau vom Mann im wesentlichen eine soziale Projektion der Selbstentfremdung ist, die biologische Unterschiede in Machtverhältnisse umsetzt. Diese Verhältnisse werden dann in sozialen Strukturen und kulturellen Erscheinungsformen totalisiert. So wird die autonome Persönlichkeit der Frau eliminiert und sie wird allein durch die Wünsche und Negationen der Männer bestimmt.

Während Rosemary Ruether im Gefolge der Befreiungstheologie Sünde weitgehend als soziale und zwischenmenschliche Entfremdung ansieht, ist ihr die Christusgestalt für die Erlösung von der Sünde kaum maßgebend. Dies ist auch nicht verwunderlich, denn, wie Letty Russell betont, Frauen stehen Jesus nicht etwa deswegen kritisch gegenüber, weil er jüdischer Herkunft war oder zu seiner Lebenszeit keine große geschichtliche Rolle spielte, sondern zuvorderst, weil er ein Mann war. ${ }^{12}$ Wie kann der Mann Jesus der Träger des Heils sein, wenn er nur die Hälfte der Menschheit repräsentiert? Eine Möglichkeit, die von den radikalsten Vertretern der Befreiung der Frauen vorgeschlagen wird, ist, nach einer weiteren Inkarnation in Form einer Frau zu suchen. Mary Daly, in Beyond God the Father

9 Vgl. Gayraud S. Wilmore, "Black Messiah: Revising the Color Symbolism of Western Christology", Journal of the Interdenominational Theological Center, Bd. II (1974), 8-18.

${ }^{10}$ So J. Deotis Roberts, "Theology of Religions: The Black Religious Heritage," Journal of the Interdenominational Theological Center, Bd. II (1974), 66.

"Vgl. Rosemary Radford Ruether, "Sexism and the Theology of Liberation. Nature, Fall and Salvation as Seen from the Experience of Women," Christian Century, Bd. XC (Dec. 12, 1973), $1224-1229$.

12 Letty M. Russell, Human Liberation in a Feminist Perspective. A Theology, Philadelphia 1974, S. 137. 
(dt.: Jenseits von Gottvater Sohn E Co. Aufbruch zu einer Philosophie der Frauenbefreiung), sieht in der Idee eines einzigartigen männlichen Heilsereignisses einen weiteren Versuch, die männliche Vorherrschaft zu legitimieren. ${ }^{13}$ Deshalb wertet sie jede christliche Lehre als doketisch, die nicht mit der Tatsache ernst macht, daß Jesus ein endliches menschliches Wesen war. Damit werden alle christologischen Formulierungen abgelehnt, die zu einer Verehrung der Person Jesu ermutigen oder eine solche reflektieren. Doch bleibt Mary Daly in gewisser Weise noch in der christlichen Tradition, wenn sie für eine Wiederkunft optiert, aber nicht des Christus, sondern getreu ihres antichristlichen, d.h. anti-männlichen Ansatzes, des Antichrists. Dieser Antichrist kann aber nach Daly nicht in der Gestalt der Maria gesehen werden, denn auch sie verkörpert nur Duldung und Unterwerfung, sondern in der Wiederkunft der Frau. Die Wiederkunft muß in der prophetischen Dimension des Symbols der Magna Mater gesehen werden, die zur Mutter Gottes verkürzt wurde. Diese Wiederkunft ist der Schlüssel zur Befreiung von Knechtschaft in den Strukturen, die menschliches Leben verhindern. Symbolisch gesprochen muß die Jungfrau »den Sohn befreien und serretten «.$^{14}$

Im Gegensatz zu Mary Daly bejahen viele Theologinnen, daß Christus die Zentralfigur des christlichen Glaubens ist und der einzigartige Vertreter der Menschlichkeit Gottes. Zwar bleibt der Anstoß an dem Mann Jesus bestehen, aber er wird nicht zum Stolperstein. Sie erkennen, wie Letty Russell betont, daß am Werk Christi nicht das wichtigste ist, daß Gott ein Mann wurde, sondern daß er ein Mensch wurde. In der androzentrischen Kultur und der patriarchalischen Religion, sagt Russell, war es vielleicht wichtig, daß Gott einen Mann sandte, damit er die Freiheit hatte, als Rabbiner zu lesen und zu lehren. ${ }^{15}$ Zudem können auch Frauen in Jesus eine einzigartige Enthüllung wahrer Menschlichkeit sehen, denn er half Männern und Frauen, ihre eigene totale Persönlichkeit zu verstehen. Indem Jesus die Möglichkeit demonstrierte, eine volle Persönlichkeit zu werden, eröffnete er diese Möglichkeit auch anderen. Falls man bei Jesus zunächst an einen Mann denkt oder an einen Juden, verfällt man wieder in den biologischen Determinismus, der Sex und Hautfarbe zu den wichtigsten Elementen einer Person erklärt. Doch ist die wichtigste Erkenntnis, daß wir Personen sind, während die biologische Differenzierung sekundär ist. So hat auch Paulus Christus als den neuen Adam verstanden, durch den das Ideal des ersten Menschen verwirklicht wurde.

Diese Verwirklichung durch Inkarnation, Tod und Auferstehung wird für uns im Glauben zugänglich. Damit wird das Symbol des neuen Men-

\footnotetext{
${ }^{13}$ Mary Daly, Jenseits von Gottvater Sohn E Co. Aufbruch zu einer Philosophie der Frauenbefreiung, übers. v. Marianne Reppekus u. Barbara Henninges, München 1980, S. 88.

${ }_{14}$ Ebd., S. 116.

${ }^{15}$ Letty M. Russell, a. a. O., S. 138.
} 
schen für uns zur Einladung, erwachsen zu werden und Verantwortung auf uns zu nehmen in unserer Rolle als Repräsentanten des neuen Menschen, um Befreiung und Segen in unser Leben und das aller anderen Menschen zu bringen. In dieser Variante der Befreiungstheologie ist Christus Vorbild und Ermöglichungsgrund des neuen Menschseins. Dabei werden die $\mathrm{Ge}-$ fahren einer solchen Weltveränderung nicht verniedlicht und auch der eschatologische Vorbehalt einer neuen Welt kann durchaus gewahrt bleiben. Die Befreiungschristologie erwächst zutiefst aus der Erfahrung der Entfremdung und Sündhaftigkeit und sieht, von einigen radikalen Ausnahmen abgesehen, Person und Werk Christi für unser Heil als konstitutiv an. Somit muß man erkennen, daß es sich bei dieser Art der theologischen Reflexion nicht um eine Modeerscheinung handelt.

Der mennonitische Theologe John Howard Yoder behauptet in seinem Buch The Politics of Jesus, daß es belanglos sei, wer Jesus war und was er tat, falls er kein ethisches Vorbild für uns ist. ${ }^{16}$ In bewußtem Gegensatz zur herkömmlichen Ethik erklärt Yoder, daß Jesus für die gegenwärtige Ethik nicht nur bedeutsam, sondern auch normativ ist. Yoder versucht zu zeigen, daß die Imperative der frühchristlichen Gemeinde großenteils aus ihrem Bekenntnis zu Christus als dem Herrn herauswuchsen und damit auch mit der Tradition in Verbindung standen, die von Jesus herkommt. ${ }^{17}$ Dabei zeigt sich in den ethischen Verhaltensmaßregeln des Neuen Testaments, daß durch das Evangelium von Christus die Christen von der Weltverhaftetheit befreit wurden, denn Christus nahm an unserer Stelle die Knechtschaft der Geschichte auf sich. Den Gläubigen wird deutlich, daß sie nicht mehr den versklavenden und entfremdenden Mächten dieser Welt unterworfen sind. Die Befreiung durch Christus bezieht sich auf jede Art von Knechtschaft und will im Einklang mit dieser radikalen Veränderung wirken. Doch im Gegensatz zu den meisten Befreiungstheologen betont Yoder, daß um des Vorbildes Christi willen diese Veränderung nicht mit Gewalt der Sozialordnung außerhalb der Grenzen der Kirche aufoktroyiert werden soll.

Die freiwillige Unterwerfung der Kirche ist als Zeugnis an die Welt verstanden. $\mathrm{Da}$ durch Auferstehung und Pfingsten das unmittelbar bevorstehende Reich Gottes zum Teil in die Geschichte eingegangen ist, kann die Kirche nun innerhalb der Strukturen der Gesellschaft das neue Leben dieses Reiches verkörpern. ${ }^{18}$ Wenn wir uns deshalb als Frau, Kind oder Sklave der Welt unterwerfen, da das dem Herrn gemäß ist, dann haben wir nicht die Radikalität des Rufes Jesu kompromittiert. Im Gegenteil, wir brauchen uns nicht mehr mit Gewalt gegen die Strukturen dieser Welt zu stellen, denn

\footnotetext{
${ }^{16}$ John Howard Yoder, The Politics of Jesus. Vicit Agnus Noster, Grand Rapids, Michigan 1972, S. 23.

17 Ebd., S. 182.

18 Ebd., S. 192.
} 
diese werden sowieso vergehen, wie Christus gelehrt und durch sein Geschick demonstriert hat. Indem wir uns völlig unterwerfen, da das in der Gesellschaft so ist, können wir ohne Ressentiment leben und zugleich Personen in sozial dominierenden Stellungen dazu anhalten, dem dominierenden Aspekt ihrer Stellen zu entsagen. Damit wird kein status quo gutgeheißen, denn durch die gehorsame Nachfolge Jesu und durch die Kraft, die von ihm und seinem Geschick ausgeht, ergibt sich eine schöpferische Umformung der Welt. Die pazifistische aber nicht quietistische Ethik Yoders ist zutiefst in Person und Werk Christi verwurzelt. Obwohl in ihr mennonitisches Gedankengut zutagetritt, sollte man sich doch fragen, ob damit nicht ein zentrales Moment aller genuinen Christologie getroffen ist.

\section{b) Von der totalen Inkarnationstheologie zur Erwartung der Wiederkunft Christi in den Wolken}

Die Blütezeit der Gott-ist-tot Theologie ist sicher vorbei. Doch wenn William Hamilton in The New Essence of Christianity feststellt ${ }^{19}$, daß man das Gefühl hat, daß sich Gott irgendwie zurückgezogen hat und daß man deswegen nicht einfach in dialektischer Weise Gott in der Zurückgezogenheit und Schwachheit findet, so hat er damit sicher eines der Grundprobleme der Christologie und der Heilswirklichkeit angerührt.

Als er darauf angesprochen wurde, daß er zu sehr das Kreuz und die Herrschaft Jesu als Erniedrigung betone, antwortete Hamilton, daß er absichtlich den Teil des christlichen Kerygmas ignoriere, der von der Himmelfahrt, der Erhöhung und dem königlichen Amt sprach. Obwohl er diese Attribute nicht ablehnen oder für falsch halten will, fragt er sich, wie und ob man sie denn heute mit konkreter Bedeutung füllen könnte. Etwas besser sieht er die Interpretationsmöglichkeit der Auferstehung Jesu. Doch auch hier sieht er die Herrschaft Jesu wie sie in der Auferstehung zutagetritt, als eine Herrschaft der Erniedrigung und des Leidens, denn auch der Auferstandene hat noch die Nägelmale an seinem Leib. ${ }^{20}$

Thomas J.J. Altizer folgt den Gedanken William Hamiltons und versucht, eine konsequent kenotische Christologie zu entwickeln. ${ }^{21}$ Für Altizer ist der Abstieg Gottes in das menschliche Fleisch endgültig und unumkehrbar. Der Tod Gottes ist ein Ereignis in der Geschichte, denn »der Tod Gottes in Christus ist eine unvermeidliche Folge des Eintritts Gottes in die Welt, des Geistes ins Fleisch; und die Verwirklichung des Todes Gottes in der Erlebnistotalität ist ein entscheidendes Indiz für die fortwährende Vorwärtsbewegung des Göttlichen, im dauernden Vollzug der Verneinung seiner partikularen und gegebenen Äußerungen des begriffenen und immer

\footnotetext{
19 William Hamilton, The New Essence of Christianity, New York 1966.

${ }^{20}$ Ebd., S. 116, Anm. 34.

21 Thomas J. J. Altizer, The Gospel of Christian Atheism, Philadelphia 1966, S. 11.
} 
voller in die Tiefen des Profanen sich hineinbegebenden Prozesses. «22 Indem sich das fleischgewordene Wort von Gott zu Jesus bewegt und seine kenotische Bewegung weitervollzieht, und von dem irdischen Jesus in den universalen Körper der Menschheit eingeht, kommt es zu einer Epiphanie Gottes in jedem menschlichen Gesicht und jeder Hand. Gott wird selbst zunichte, damit wir vergöttlicht werden. Das Heilsgut wird immanent als die Epiphanie einer neuen Welt und der Wiederentdeckung des Göttlichen gesehen.

Angesichts einer Welt, die immer mehr Züge des Dämonischen manifestiert, wirkt aber solch ein Hegelscher Pantheismus Straußscher Prägung nicht recht überzeugend. Deshalb scheint heute in weiten Bevölkerungskreisen, besonders gefördert durch das Dallas Theological Seminary unter John F. Walvoord und J. Dwight Pentecost, bis ins einzelne detaillierte, jenseitige Heilserwartung immer größeres Interesse zu finden. Man überläßt die Welt dem Verderben und flüchtet sich ins Jenseits.

So wird gelehrt, daß die Gläubigen vor dem Einsetzen der eschatologischen Wehen zu dem wiederkehrenden Herrn entrückt werden, ehe er sein Tausendjähriges Reich auf Erden aufrichtet. In einer bisher 13 Folgen umfassenden Serie, "Posttribulationism Today «, in seiner Hauszeitschrift Bibliotheca Sacra ${ }^{23}$, sagt John F. Walvoord, daß die Hoffnung für die Gläubigen zunächst darin besteht, daß sie im wörtlichen Sinne den endzeitlichen Wehen enthoben werden. Die Gläubigen sehen damit nicht so sehr der Auferstehung nach den Martyrien entgegen, sondern dem Kommen Christi und der Enthüllung der Ehre Gottes, während sie noch auf Erden leben. ${ }^{24}$

Wenn wir uns kurz der Christologie Hal Lindseys zuwenden, der am Dallas Theological Seminary studierte und durch sein Buch The Late Great Planet Earth (dt.: Alter Planet Erde wohin?) plötzlich zu einem populären Exponenten biblizistischer, prophetischer Spekulation wurde, so wollen wir hier nur auf sein Buch The Liberation of Planet Earth eingehen. ${ }^{25}$ Wichtig ist für Lindsey, daß durch das Werk Christi am Kreuz der geistliche Tod für immer überwunden wurde, der jeden Menschen bedroht. Durch seinen Tod hat uns Christus mit Gott versöhnt und Gottes Heiligkeit und Gerechtigkeit Genüge getan. Durch die Rechtfertigung erreichen wir Frieden mit Gott (Röm 5:1), so daß wir auch in Frieden mit uns selbst

${ }^{22}$ Ders., ...daß Gott tot sei. Versuch eines christlichen Atheismus, übers. v. Fritz Dumermuth, wiss. überarb. v. Wilhelm Hüffmeier, Zürich 1968, S. $126 \mathrm{f}$.

${ }^{23}$ Vgl. John F. Walvoord, "Posttribulationism Today" (13 T.), Bibliotheca Sacra, Bd. CXXXI. (Jan.-Mar., 1975) bis Bd. CXXXIV (Jan.-Mar., 1978).

24 Ders., "Posttribulationism Today, Teil 13: Pretribulationism as the Alternative to Postribulationism," a. a. O., Bd. CXXXIV (Jan.-Mar., 1978), 20.

${ }^{25} \mathrm{Vgl}$. Hal Lindsey u. Carole C. Carlson, Alter Planet Erde wohin? Im Vorfeld des Dritten Weltkrieges, übers. v. Martin Schneider, Wetzlar 1971; u. Hal Lindsey, The Liberation of Planet Earth, Grand Rapids, Michigan 1974. 
und den Mitmenschen leben können. Wir stehen dann in der Gnade Gottes, die jede Werkgerechtigkeit zunichtemacht und sind jeder Verurteilung durch Gott enthoben (Röm $8: 1$ ). Wir brauchen uns selbst nicht mehr zu verdammen und dürfen auch anderen gegenüber in Offenheit und Vergebung leben. Unser Verhältnis zu uns und unseren Mitmenschen wird somit von der Vergebung Christi her gesehen. Natürlich sind wir immer noch von unserer alten, sündhaften Natur umgeben, vom Gesetz und vom Satan. Obwohl sie nicht im Nichts verschwinden, haben sie durch den Opfertod Christi keinen Anspruch auf uns, außer wir würden unsere neugewonnene Freiheit in Christus aufgeben. Wenn wir so zur Freiheit der Kinder Gottes geistig wiedergeboren werden, sind wir fähig, geistige Dinge Gottes zu verstehen. ${ }^{26}$ Lindsey versichert uns, daß wir in diesem Leben bestehen können, denn Gottes Licht erleuchtet unsere Wege. Die Segensfrüchte der Rechtfertigung und Heiligung erstrecken sich auf das Heil hier und im Jenseits. Unsere ganze Persönlichkeit wird neu orientiert und wir werden selbstverständlich am Ende das Reich Gottes ererben.

Wie das Heil aussieht, das uns Christus bereitet, wird von James Montgomery Boice in seinem Buch The Last and the Future World in allen Einzelheiten beschrieben. Nachdem er drastisch die eschatologischen Wehen ausmalte, wie etwa zunehmende Polarisierung der amerikanischen Gesellschaft, Legalisierung vieler sündhafter Praktiken wie Abtreibung, Pornographie, Rauschgift und Homosexualität, Erwachen eines neuen Zentrums der politischen Macht im Osten und auch in Europa, fragt er: "Wie können wir der biblischen Ermahnung folgen, uns nicht zu ängstigen? " Die Lösung liegt darin, daß wir Jesus vertrauen. »Er ist Gott. In ihm, dem Herrn der Geschichte, finden wir den, der allein unser Beschützer ist. «" Das Heil, das uns hier verheißen wird, berührt also nicht die sozialen Bezüge der Gesellschaft oder das Verhältnis zwischen Mensch und Natur. Es wird im Gegensatz dazu als Heil definiert, das nur zwischenmenschliche und hier meist familiäre Beziehungen betrifft. Es erlaubt mir, mich gut zu fühlen, denn Gott liebt mich und deswegen kann ich z. B. auch meinen Ehegatten lieben. Bei Boice kann die neue Welt weder proleptisch vorweggenommen werden, noch ist der Realismus der Anfechtung gewahrt. Wiederum wird man jeglicher Verantwortung für die Welt enthoben, denn die Heilserfahrung erstreckt sich nur auf die private Sphäre des Menschen. Im Gegensatz zur Befreiungstheologie lebt der Glaube hier nur auf die jenseitige Heilsvollendung hin. Aber er empfängt keine Impulse von diesem jenseitigen Ziel für eine kreative Veränderung der Welt.

${ }^{26}$ Hal Lindsey, The Liberation of Planet Earth, S. 194.

27 James Montgomery Boice, The Last and the Future World, Grand Rapids Michigan 1974, S. 131 . 


\section{Die Prozeßchristologie als Beitrag der nordamerikanischen Forschung zur Interpretation der Wirklichkeit}

Wenn wir nun auf die Prozeßchristologie zu sprechen kommen, bewegen wir uns in einem völlig anderen Kreis theologischer Reflexion. Wenn man die Christologie eines $\mathrm{Hal}$ Lindsey als die dominante Laientheologie charakterisieren will, die aber auch unter den Theologen verschiedener fundamentalistisch und erwecklich ausgerichteter Denominationen groBen Rückhalt hat, ist die Prozeßtheologie weithin nur in akademischen Kreisen beheimatet. Sie stützt sich auf den anglo-amerikanischen Mathematiker und Philosophen Alfred North Whitehead, der hier ähnlich als unfreiwilliger Kirchenvater fungiert wie ehemals Martin Heidegger in Deutschland. Die Prozeßtheologen sind sich einig, daß Whiteheads Philosophie die beste Grundlage für eine zeitgemäße Interpretation des christlichen Glaubens abgibt. Das Zentrum der Prozeßtheologie ist Claremont mit David Griffin als Direktor des Instituts für Prozeßstudien und John B. Cobb als Professor für Systematische Theologie. Doch gibt es Prozeßtheologen in fast jeder Graduate School und allmählich auch an kirchlichen Seminaren. Für lange Zeit war die Frage ungelöst, welchen Stellenwert bei Prozeßtheologen Christus hat, die zentrale Figur des christlichen Glaubens, denn Whitehead hatte nicht viel über ihn zu sagen. Nachdem in den letzten fünf Jahren drei Prozeßchristologien erschienen sind, kann man wenigstens eine vorläufige Antwort geben.

\section{a) Christologie als Funktion der Ekklesiologie (Lewis Ford)}

Lewis Ford brachte 1978 ein Buch heraus mit dem Titel The Lure of God. A Biblical Background for Process Theism, in dem er der Prozeßchristologie einige Kapitel widmete. Prozeßtheismus, behauptet er, kann nicht die apokalyptische Spekulation der Bibel teilen, denn er versteht die Zukunft als etwas, das organisch aus seiner Vergangenheit herauswächst. ${ }^{28}$ So sollten wir die Vollendung der Welt nicht als ein futuristisches Ereignis verstehen, durch das Gott die Welt berührt, da ohne Welt Gott weder Vergangenheit noch Zukunft hat, sondern reine Gegenwart ist. "Nichtzeitlich schafft Gott sich selbst als die Inansichtnahme [envisagement] der Unendlichkeit aller reinen Möglichkeiten ${ }^{29}{ }^{29}$ Wie die Welt Zukünftigkeit von Gott bekommt, erhält Gott von der Welt Vergangenheit.

Die wechselseitige Abhängigkeit von Gott und Welt hat eminente Bedeutung für die Christologie, denn das ständige Problem der klassischen Christologie, wie eine Person vere homo et vere deus sein kann, wird nach Ford durch die Whiteheadschen Kategorien überwunden. Nach White-

${ }^{28}$ Lewis S. Ford, The Lure of God. A Biblical Background for Process Theism, Philadelphia 1978, S. 24.

${ }^{29}$ Ebd., S. 40. 
head, behauptet Ford, sind Ereignisse und Begebenheiten primär, während die substantielle Person davon abgeleitet wird. Darüber hinaus kann ein konkretes, tatsächliches Ereignis weder als reines Werk Gottes, noch als rein menschliches Werk oder als das einer anderen Kreatur verstanden werden. ${ }^{30}$ Durch sein Mitfühlen mit dem Menschen Jesus, behauptet Ford, wurde Gott nicht ein Mensch, sondern er wurde menschlich, indem er gewisse menschliche Prädikate erfuhr, wie etwa die Sorge um einen geliebten Menschen und die Angst, von einem Geliebten entfremdet zu sein. Auch meint Ford, daß für die frühe Kirche die Zweinaturenlehre oder die Inkarnation nicht so wichtig waren wie das Bekenntnis zu dem Auferstandenen, zu Jesus als dem Herrn. ${ }^{31}$

Bei Ford wird die Auferstehung des Leibes, die er für zentral hält, mit der Auferstehung des Leibes Christi als Kirche identifiziert, die durch die dynamische Kraft des Geistes Christi geleitet wird. Die Auferstehung wird daher als die Inkarnation des göttlichen Wortes angesehen, das an die menschliche Situation gerichtet ist. Damit ist die Inkarnation nicht allein und nicht primär im Leben Jesu lokalisiert, obwohl sie sich nicht unabhängig davon ereignen konnte, sondern die Inkarnation ist das ganze Ereignis der Entstehung des Leibes Christi. Die Inkarnation setzt ein menschliches Leben voraus, das für die Absichten Gottes total offen ist, ein Leben, dem als von Gott kommend andere total vertrauen konnten.

Da Inkarnation gewissermaßen mit der Auferstehung gleichgesetzt wird, ist letztere ein wesentlicher Bestandteil des christlichen Glaubens, auch wenn, wie wir später sehen werden, David Griffin das Gegenteil behauptet. Die Auferstehung ist die Grundlage, die uns erkennen läßt, was Gott in Christus bewirkt hat. ${ }^{32}$ Durch den Akt des Verlassenseins, den Jesus in seinem Tod fühlte, wurde die Auferstehung in naher Zukunft angeregt. Ford gesteht ein, daß der Tod Jesu für Gott eine Niederlage durch die Mächte des Bösen war, da er Jesus nicht in seiner Verlassenheit am Kreuz trösten konnte. Doch siegte Gott über das Böse und erweckte Jesus als den Christus. Aber es gibt keine metaphysischen Garantien, daß die Mächte des Bösen nicht Gott wieder überwältigen könnten, Gott ist jedoch bis jetzt siegreich geblieben und was er bis jetzt getan hat, kann er wieder tun.

Lewis Ford versichert uns abschließend: „Prozeßtheismus ist ein natürlicher Bundesgenosse der biblischen Geschichte, denn der Prozeß ist abstrahierte Geschichte. Prozeßtheismus kann zeitgemäße Kategorien zur Verfügung stellen, durch die wir uns diese alte Literatur aneignen können. Die biblische Tradition wiederum kann jene konkreten Einzelheiten abgeben, durch die unser Leben endgültigen Sinn bekommt. ${ }^{33}$ Es ist nicht nur

\footnotetext{
30 Ebd., S. 50.

31 Ebd., S.67.
}

32 Ebd., S. 79.

33 Ebd., S. 135. 
die Frage an Ford zu richten, ob es biblisch zu vertreten ist, daß Christus in und als Kirche auferstanden ist, sondern auch, was in seiner Interpretation das Geschick Jesu als Christus zur Daseinsbewältigung und Weltveränderung beiträgt. Können wir nach seiner Interpretation darauf vertrauen, daß Gott eine gerechtere oder neue Welt schafft, wenn doch die Mächte des Bösen Gott wieder überwältigen können? "Das Wörtlein “, das nach Luther diese Mächte fällen kann, ist bei Ford zu wenig in seiner ganzen Machtfülle und Tragweite erkannt.

\section{b) Christologie obne Auferstehung (David Griffin)}

Ähnlich wie Ford bekennt David Griffin, daß die Prozeßphilosophie der Vernunft entspricht und uns überzeugt, daß die wesentlichen Bestandteile des christlichen Glaubens wahr sind. Zentral für den Glauben ist dabei die Erkenntnis der selbstenthüllenden Tätigkeit eines persönlichen Gottes. Griffin wehrt sich gegen atheistische Christologen, die Jesu Bedeutung wesentlich in der ethischen Sphäre sehen, denn wenn sich unser Glaube an die letztgültige Wirklichkeit der Dinge nicht von Jesus ableitet, werden auch unser Charakter und unser Verhalten bald aufhören, von ihm entscheidend beeinflußt zu sein. ${ }^{34}$ Griffin bemüht sich deswegen zu zeigen, daß Jesus Gottes entscheidende Offenbarung ist. Dabei stellt er fest, daß der historische Jesus zentral für die ganze christologische Aufgabe ist, denn normative oder präskriptive Feststellungen über Jesus als den Christus müssen immer eine Lehre von der Person Jesu voraussetzen..$^{35}$

David Griffin untersucht zunächst Jesu Schau der Wirklichkeit, wie sie sich besonders im Begriff des Reiches Gottes konkretisiert. Jesus sah Gottes Walten in der Gegenwart in fragmentarischer Weise als Antizipation dessen, was für die Zukunft beabsichtigt war. Obwohl Griffin zugibt, daß die volle Ankunft des Reiches Gottes von Jesus in völlig supra-naturalistischer Weise verstanden wurde, versichert er uns, daß wir das heute nicht mehr nachzuvollziehen brauchen. Eine endzeitliche Lösung braucht nicht daraus zu folgen, daß Jesus Gottes Offenbarung war. ${ }^{36}$ Doch ist wichtig, daß nach Jesu Schau es das Ziel Gottes ist, daß alle Kreatur in der Zukunft völlig seinem Willen folgt und das Dasein unqualifiziert als gut erfährt. Jesus, wie er sich uns in Wort und Tat darstellt, war Gottes höchste Tat, denn er zeigte uns eine Wirklichkeitsschau, die eine Schau von Gottes Wesen einschließt. So war Jesus nicht nur vorbildlicher Mensch, an dem sich alle orientieren können, sondern "die Ziele, die Jesus gegeben wurden und die während seines aktiven Lebens von ihm verwirklicht wurden, waren von solcher Art, daß die grundlegende Wirklichkeitsschau, die in seiner Bot-

${ }^{34}$ David R. Griffin, A Process Christology, Philadelphia 1973, S. 17.

${ }^{35}$ Ebd., S. 199.

${ }^{36}$ Ebd., S. 205. Griffin beruft sich hier auf Adolf von Harnack und lehnt Wolfhart Pannenbergs Lösung ab. 
schaft und seinem Wirken enthalten war, der höchste Ausdruck von Gottes ewigem Charakter und Zweck war. « ${ }^{37}$ Damit ist klar, daß die Bedeutung Jesu weder von Jesus allein abhing, noch von Gott, sondern von der Art des Zusammenwirkens beider. Indem Jesus das besondere Ziel Gottes für sich verwirklichte, drückte er Gottes Ziel für die ganze Schöpfung aus ${ }^{38}$ Doch sagt Griffin abschließend, daß wahre Offenbarung Gottes uns nicht nur über dessen Tätigkeit informiert, so daß sie nur unser Verhältnis zu Gott ändern kann. Offenbarung bringt auch eine Änderung des Einwirkens Gottes auf uns Menschen mit sich. Gott kann damit nicht mehr ungeschehen machen, wie er sich uns in seiner Offenbarung erschlossen hat.

Es ist wichtig zu notieren, daß Griffin dem Tod Christi kaum Beachtung schenkt und auch die Auferstehung Christi absichtlich ausklammert. Obwohl er in seinem Buch God, Power and Evil. A Process Theodicy zugibt, ${ }^{39}$ daß die in der Auferstehung Christi begründete Hoffnung auf unsere eigene Auferstehung den Sinn für die Wichtigkeit des menschlichen Lebens vertieft, und die Hoffnung auf eine zukünftige, höhere Form der Existenz in diesem gegenwärtigen Leben mehr Sinn geben kann, möchte er doch die Entscheidung für einen Glauben an die Auferstehung der persönlichen Einstellung überlassen, da sie zur Natur des Glaubens nichts beiträgt. Auch die Auferstehungserscheinungen sind für Jesu Bedeutung nicht konstitutiv. Griffin behauptet, daß die Auferstehung Jesu heute kaum noch als Grundlage des eigenen Glaubens an seine besondere Bedeutung dient. Meist wird sie dann nur angenommen, wenn die besondere Bedeutung Jesu bereits erkannt wurde. So kann sich für Griffin der christliche Glaube ohne Auferstehung Jesu und ohne Hoffnung auf eine persönliche Auferstehung oder auch auf eine persönliche Unsterblichkeit konstituieren.

\section{c) Christologie als schöpferische Umformung [creative transformation]} (Jobn B. Cobb Jr.)

Der wohl bekannteste Exponent der amerikanischen Prozeßtheologie, John B. Cobb Jr. beschreibt in seinem umfangreichen Buch Christ in a Pluralistic Age Christus als den Weg, der keine anderen Wege ausschließt. ${ }^{40}$ Damit ist bereits die universalistische Tendenz der Denkbemühungen Cobbs angezeigt. In der christlichen Tradition, behauptet Cobb, wird die transzendente Wirklichkeit, die in ihrer fleischgewordenen Form Christus genannt wird, als Logos bezeichnet.

Christus ist der fleischgewordene Logos. Der Logos ist das kosmische Ordnungsprinzip, der Bedeutungsgrund [ground of meaning], und die Quelle aller Zwecke der Welt. Whitehead nannte diese transzendente

${ }^{37}$ Ebd., S. 218.

${ }^{38}$ Ebd., S. 220.

39 Ders., God, Power and Evil: A Process Theodicy, Philadelphia 1976.

40 John B. Cobb, Jr., Christ in a Pluralistic Age, Philadelphia 1975, S. 22. 
Zielquelle des Neuen das Prinzip der Konkretisierung, das Begrenzungsprinzip, das Organ der Neuheit und Gott in seiner ursprünglichen Natur. Cobb versucht nun diese Gedanken Whiteheads mit denen der klassischen Logostheologie zu verbinden, indem er - ähnlich wie Tillich - die Identität des fleischgewordenen Logos mit dem universalen Logos betont. Wenn wir statt Logos Christus sagen, erkennen wir, daß der kosmische Logos Liebe ist. Wenn der Glaube die ursprüngliche und richtige Haltung Gott gegenüber ist, dann ist der Glaube die Vernunft in ihrem Kampf mit dem Aberglauben. Er ist Teilnahme an einer schöpferischen Umformung. ${ }^{41}$

Die Frage erhebt sich, wie die schöpferische Umformung, die Cobb als den Kern der Logoschristologie ansieht, auch aus Jesus von Nazareth erhoben werden kann. Cobb will zunächst das Verhältnis zwischen dem, was man von Jesus weiß, und der schöpferischen Umformung [creative transformation] bestimmen, die Christus genannt wird. Cobb stellt fest, daß sich aus den ältesten Traditionsgeschichten glaubwürdige Information über Jesus und seine Verkündigung gewinnen läßt. ${ }^{42}$ Natürlich gibt es für die Botschaft Jesu Parallelen in der jüdischen Literatur seiner Zeit. Aber "Jesus radikalisierte die Bedeutung dieser Ideen und formte das, was er von anderen übernahm, so um und trug sie in solcher Weise vor, daß ihre konkrete Bedeutung für ihn und seine Hörer neu war. « $^{43}$ So können wir in Jesus schon den Christus sehen, nämlich die schöpferische Umformung.

Die Frage muß jedoch gestellt werden, wieso diese schöpferische Umwandlung, die man auch in vielen anderen Gestalten sehen könnte, für uns heute von Bedeutung ist. Cobb stellt Jesus nicht nur neben andere, sondern behauptet, daß in ihm auch eine schöpferische Umwandlung anderer stattfand. So riefen etwa seine Worte und Taten seine Zuhörerschaft vor die Entscheidung, ob sie ihm nachfolgen oder ihn ablehnen sollten. Auch Paulus betont, daß die auf Christus Getauften Christus anziehen sollen und damit ihm gleich werden sollen.

Wer war nun dieser Jesus, der als Christus bezeichnet wird und von dem solche Impulse ausgehen? Zunächst stellt Cobb fest, daß er in jeder Hinsicht ein Mensch war. ${ }^{4}$ Aber zugleich wurde in ihm der Logos völlig Fleisch. Das heißt, die Inkarnation des Logos fand in ihm nicht wie in jedem anderen Wesen statt, sondern in solcher Weise, daß der Logos mit dem Selbst Jesu zusammenfiel. Die Verkörperung dieser Existenzstruktur erklärt damit Jesu Gewißheit und Autorität. ${ }^{45}$ Wenn wir Jesus so verstehen, daß er im Vollsinne Mensch war und Gott in ihm gleichzeitig wahrhaft innewohnte, dann ist es möglich, daß die Gedanken Gottes uns in dem begegnen, was wir von Jesus wissen. Wenn wir in Jesus Gottes Immanenz und sein Handeln erkennen, dann können wir von Jesus lernen, wie Gott

\footnotetext{
4 Ebd., S. 93.

42 Ebd., S. 101.

${ }^{43}$ Ebd., S. 107.
}

4 Ebd., S. 134.

4 Ebd., S. 138. 
ist. Jedoch dürfen wir Gott nicht mit Jesus identifizieren, denn Immanenz ist nicht Identität. Ontologische Fragen, erklärt Cobb, können nicht durch das Studium Jesu entschieden werden. Aber die Weise, wie sich Gott zur Welt verhält, sollte in Jesus sichtbar werden. Durch die Präsenz des Logos in Jesus ist auch die Frage der Freiheit Jesu entschieden, denn je größer die effektive Präsenz des Logos ist, desto größer ist die menschliche Freiheit in der Entscheidung dessen, was tatsächlich erreicht werden soll.t

Wenn wir schließlich Cobb nach dem Heilsgut fragen, so verweist er auf das Reich Gottes und die Auferstehung der Toten. Doch schränkt er sofort ein, daß weder Jesus noch Whitehead das Reich Gottes mit spezifischen sozial-politischen Programmen verbanden. Aber nach Jesus mußte durch das Reich Gottes die Geschichte verändert werden, so daß Gerechtigkeit herrschen konnte. Jesu Schau der Geschichte rief in der Geschichte Reformen und Revolutionen hervor und bringt auch heute noch die gegenwärtige Ungerechtigkeit und das Elend der Gesellschaft ans Licht und untermauert die Forderungen der Schwachen und Machtlosen." Doch was ist das Ziel der Geschichte? Cobb lehnt als Erfüllung eine ewige Selbstidentität der Person ab. Die engen Grenzen des Selbst sind im Reich Gottes verschwunden. In das Reich wird all das Eingang finden, was in solch einem vervollkommnenden System sein kann. Unsere Leiden, Entbehrungen, Versagen, Triumphe und Unmittelbarkeiten der Freude werden alle durch das rechte Gefühl in die Harmonie des universalen Gefühls eingewoben. ${ }^{48}$ Unser individuelles Selbst aber wird im Reich Gottes umgeformt und in Konformität mit der endgültigen, absoluten Weisheit gereinigt. Dieses Weben und Eingliedern ist ein ständiger (kosmischer) Prozeß, da neue, zeitliche Ereignisse sich in die Einheit der Unmittelbarkeit aller Ereignisse des Reiches einfügen.

Diese Schau steht natürlich im Gegensatz zu der traditionellen Lehre von der Auferstehung der Toten. Doch Cobb findet, daß durch diese Lehre die einzelne Persönlichkeit zu viel Gewicht erhält und die kosmische und menschliche Geschichte zugunsten der erhofften Neuschöpfung abgewertet wird. ${ }^{49}$ Für Cobb wird die Linie zwischen dem transzendenten Reich und der irdischen Geschichte nicht als unüberbrückbar angesehen, denn die Wirklichkeit des Reiches geht in die Wirklichkeit der Welt ein und umgekehrt. Während dieser Ansatz uns sicherlich von einer manichäischen Verwerfung der Welt bewahrt, wird aber doch auch die Grundschwäche der Prozeßchristologie deutlich, die Verharmlosung des Bösen, und das Desinteresse an der einzelnen Person.

Was ergibt sich nun aus diesen verschiedenartigen nordamerikanischen Arbeiten zur Christologie? Man kann das in zwei Punkten zusammenfassen:

${ }^{46}$ Ebd., S. 172.

47 Ebd., S. 228.
${ }^{48}$ Ebd., S. 248.

49 Ebd., S. 250. 
1. Durch den Pluralismus der nordamerikanischen Gesellschaft ist eine Vielfalt der Christologien unabdingbar.

2. Innerhalb dieser Vielfalt sind bei der Bestimmung des Heilsgutes zumindest zwei Phänomene charakteristisch:

a) Das praxisbezogene Denken der Amerikaner sträubt sich gegen ein rein vergeistigtes Heilsgut. Es wird entweder auf die sozioökonomischen Bedingungen, auf das interpersonale Verhältnis der Einzelnen, oder auf die Gesamtheit der Menschen bezogen.

b) Der Kulturoptimismus der Amerikaner drückt sich auch darin aus, wie der Heilserwerb gedacht ist. Obwohl zumeist die Tiefe der Sündhaftigkeit der Menschheit nicht verschwiegen wird, ist man geneigt, das Heil mehr durch die von der Person Christi ausstrahlende Kraft gewirkt zu sehen als durch das, was durch das Werk Christi bewirkt wurde. So hat H. Richard Niebuhr immer noch recht, wenn er einst über den nordamerikanischen Kontext sagte: "Ein Gott ohne Zorn leitete Menschen ohne Sünde in ein Reich ohne Gericht durch die Vermittlung eines Christus ohne Kreuz. ${ }^{50}$

Doch greift die nordamerikanische christologische Forschung einige Momente auf, die auch für die deutsche Theologie von Bedeutung sind:

1. Der Pluralismus Nordamerikas zeigt sich etwa nicht primär darin, daß die verschiedensten christologischen Entwürfe gleichberechtigt nebeneinander gelten gelassen werden, sondern, wie bei der Christologie der Schwarzen oder der Frauen, daß die Christologie die vorhandenen sozio-kulturellen Kontexte durchdringen muß, um darin wirksam zu werden. Daß damit die Gefahr christologischer Domestizierung gegeben ist, wird an unserem kraftlosen weißen Christus immer deutlicher.

2. Die Relevanz der Christologie wird nicht zuletzt dadurch bestimmt, daß das Heilsgut auch auf die konkreteren Lebenssituationen, etwa die sozio-ökonomischen Bedingungen oder zwischenmenschliche Probleme bezogen wird. Daß es damit zu keinem politischen oder gruppendynamischen Programm entarten darf, sollte durch das Caveat des paulinischen "noch nicht " ständig neu eingeschärft werden.

\section{SUMMARY}

This review of Christological research in present-day North American theology presents and analyzes examples of black christology (James H. Cone), feminist christology

so H. Richard Niebuhr, Der Gedanke des Gottesreichs im Amerikanischen Christentum, dt. Ausg. v. Richard M. Honig, New York 1948, S. 140. 
(Rosemary Radford Ruether, Mary Daly, Letty Russell), Mennonite christology (John Howard Yoder), death-of-God christology (William Hamilton, Thomas J. J. Altizer), Millennial christology (Hal Lindsey), and process christology (Lewis Ford, David Griffin, John B. Cobb, Jr.).

It is our intention to present an overview of the diversity of contemporary christological positions while at the same time asking whether there are any common trends. Two trends can be discerned: An obvious pluralism, resulting from an increasing indigenization of Christ in a pluralistic society, and a peculiar description of the content of salvation. This content must be related to everyday life, whether on a personal, societal, or global level. It is especially the power of Jesus' personality which is the mediator of the content of salvation rather than work of Christ.

An extensive bibliography is appended.

\section{ERGÄNZENDE BIBLIOGRAPHIE (1968-1982) zusammengestellt von Jon $M$. Temme}

Altizer, Thomas J.J. ... daß Gott tot sei. Versuch eines christlichen Atheismus. Übers. v. Fritz Dumermuth, wiss. überarb. v. Wilhelm Hüffmeier. Zürich 1968.

Aldwinckle, Russell F. More Than Man. A Study in Christology. Grand Rapids, Mich. 1976. Anderson, Gerald H. \& Stransky, Thomas F. Christ's Lordship and Religious Pluralism. Maryknoll 1981.

Barth, Hans Martin. "Tod-Gottes-Christologie. Der christologische Ansatz der nordamerikanischen Tod-Gottes-Theologie*. Kerygma und Dogma, XVII (4/1971), 258-272.

Beeck, Frans Jozef van. Christ Proclaimed. Christology as Rhetoric. New York 1979.

Bouyer, Louis. The Eternal Son. trs. from Fr. by Simone Inkel and John F. Laughlin. Our Sunday Visitor, 1980.

Braaten, Carl. "Who Do We Say That He Is? On the Uniqueness and Universality of Jesus Christ". Occasional Bulletin of Missionary Research, IV (Jan., 1980), 2-8.

Brock, Rita. "A Feminist Looks at Christology". Encounter, XLI (Aut., 1980), 319-331.

Brown, Robert McAfee. "Who Is This Jesus Christ Who Frees and Unites?". Mid-Stream, XV (April, 1976), 129-145.

Carpenter, James C. "The Christology of John Cobb". Process Studies, VI (Summer, 1976), $103-115$.

Cleage, Albert B., Jr. The Black Messiah. New York 1968.

- "The Black Messiah and the Black Revolution". In: Quest for a Black Theology, hg. v. James J. Gardiner u. J. Deotis Roberts. Philadelphia 1971, S. 1-21.

Cobb, John B., Jr. Christ in a Pluralistic Age. Philadelphia 1975.

- "The Finality of Christ in a Whiteheadian Perspective". In: The Finality of Christ, hg. v. Dow Kirkpatrick. Nashville, Tennessee 1966, S. 122-154.

- "Response to Ogden and Carpenter". Process Studies, VI (Summer, 1976), 123-129.

- "A Whiteheadian Christology". In: Process Philosophy and Christian Thought, hg. v. Delwin Brown, Ralph E. James, Jr., u. Gene Reeves. Indianapolis 1971, S. 382-398.

Cone, James H. A Black Theology of Liberation. Philadelphia 1970, bsd. S. 197-227.

- God of the Oppressed. New York 1975, bsd. S. 108-137.

- Schwarze Theologie. Eine christliche Interpretation der Black-Power-Bewegung, mit einem Nachw. v. Frederich Herzog u. ausgew. Dokumenten. Übers. v. Ulrich G. Fick. München 1971.

- "Schwarze Theologie im Blick auf Revolution, Gewaltanwendung und Versöhnung". Evangelische Theologie, XXXIV (1/1974), 4-16.

- "Who is Jesus Christ For Us Today?". Christianity and Crisis, XXXV (April 14, 1975), 81-85. 
Cook, Michael L. The Jesus of Faith: A Study in Christology. New York 1981.

Coventry, John. Faith in Jesus Christ. Minneapolis 1980.

Daly, Mary. Jenseits von Gottvater Sohn E Co. Aufbruch zu einer Philosophie der Frauenbefreiung. Übers. v. Marianne Reppekus u. Barbara Hennings. München 1980.

Deloria, Vine, Jr. God is Red. New York 1973.

Dawe, Donald G. "Christology in Contemporary Systematic Theology". Interpretation, XXVI (July, 1972), 259-277.

Driver, Tom F. Christ in a Changing World. Toward an Ethical Christology. New York 1981.

Eichelberger, William L. "A Mytho-Historical Approach to the Black Messiah". Journal of Religious Thought, XXXIII (Spr.-Sum., 1976), 63-71.

Elizondo, Virgilio. Mestizaje. The Dialectic of Cultural Birth and the Gospel. 3 Bde. San Antonio, Texas 1978, bsd. T.3: "The Breakthrough Violence, Jesus the Liberator", Bd. II: 401-544.

Ford, Lewis S. The Lure of God: A Biblical Background for Process Theism. Philadelphia 1978.

- "The Possibilities for Process Christology". Encounter, XXXV (Aut., 1974), 281-294.

- "The Power of God and the Christ". In: Religious Experience and Process Theology. The Pastoral Implications of a Major Movement, hg. v. Harry James Cargas u. Bernard Lee. New York 1976, S. 79-92.

- "The Resurrection as the Emergence of the Body of Christ". Religion in Life, XLII (Winter, 1973), 466-477.

Frankenmolle, Hubert. Jesus Christ. His Kingdom, His Promise. New York 1982.

Frei, Hans W. The Identity of Jesus Christ. The Hermeneutical Bases of Dogmatic Theology. Philadelphia 1975.

Galot, Jean. Who is Christ? Chicago: Franciscan Herald Press, 1981.

Griffin, David R. "Essential Elements of a Contemporary Christology". Encounter, XXXIII (Spring, 1972), 170-184.

- A Process Christology, Philadelphia 1973.

Hall, Thor. The Evolution of Christology. Nashville 1982.

Idinopulos, Thomas A. "A Critical Appraisal of Differing Process Christologies”. Encounter, XXXVII (Spring, 1976), 211-215.

Johnson, Joseph A., Jr. The Soul of the Black Preacher. Philadelphia 1971, bes. S.60-107.

Mackey, James P. Jesus: Der Mensch und der Mythos. Eine zeitgemäße Christologie. Übers. v. Ulrich Hühne, München 1981.

McBrien, Richard. Catbolicism. 2 vols. Minneapolis 1980.

Milet, Jean. God or Christ? The Excesses of Christocentricity. New York 1981.

Miller, David L. Christs. Meditations on Archetypal Images in Christian Theology. New York 1981.

Moyd, Olin P. Redemption in Black Theology. Valley Forge, PA 1979, bsd. S. 131-150.

Murray, Pauli. "Black Theology and Feminist Theology: A Comparative View". Anglican Theological Review, LX (Jan., 1978), 3-24.

Ogden, Schubert M. "Christology Reconsidered: John Cobb’s 'Christ in a Pluralistic Age”". Process Studies, VI (Summer, 1976), 116-122.

- "Point of Christology". Journal of Religion, LV (October, 1975), 375-395.

O'Grady, John F. Models of Jesus. Garden City, N. Y. 1981.

Pawlikowski, John T. Christ in the Light of the Jewish-Christian Dialogue. Ramsey, NJ 1982.

Richard, Lucien. O.M.I. What Are They Saying about Christ and World Religions? Ramsey, NJ.; 1981.

Roberts, J. Deotis. "Contextual Theology: Liberation and Indigenization". Christian Century, XCIII (Jan. 28, 1976), 64-68.

- "Black Theology in the Making". Review and Expositor, LXX (Summer, 1973), 321-330.

- Liberation and Reconciliation: A Black Theology. Philadelphia 1971, bsd. S. 130-155.

Ruether, Rosemary Radford. To Change the World. Christology and Cultural Criticism. New York 1981. 
- Liberation Theology. Human Hope Confronts Christian History and American Power. New York 1972.

- New Woman, New Earth. Sexist Ideologies and Human Liberation. New York 1975.

- "What Do the Scriptures Say: The Sexuality of Jesus". Christianity and Crisis, XXXVIII (May 29, 1978), 134-137.

Rupp, George. Christologies and Cultures. Toward a Typology of Religious Worldviews. The Hague 1974.

Russell, Letty. Human Liberation in a Feminist Perspective - A Theology. Philadelphia 1974. Shea, John. The Challenge of Jesus. New York 1982.

Sittler, Joseph. "Scope of Christological Reflection". Interpretation, XXVI (July, 1972), 328-337.

Skinner, Tom. How Black is the Gospel? Philadelphia 1970, bsd. S. 92-104.

Starkloff, Carl F., People of the Center: American Indian Religions and Christianity. New York 1974.

Suchocki, Marjorie. God-Christ-Church. A Practical Approach to Process Theology. New York 1982.

TeSelle, Eugene. Christ in Context. Divine Purpose and Human Possibility. Philadelphia 1975. Thompson, Marjorie. "God, Woman, Man: Reflections of Mary and Jesus". Worship, LV (May, 1981), 327-343.

Wilmore, Gayraud S. "The Black Messiah: Revising the Color Symbolism of Western Christology". Journal of the Interdenominational Theological Center, II (Fall, 1974), 8-18.

Yoder, John Howard. The Politics of Jesus. Vicit Agnus Noster. Grand Rapids, Michigan 1972. 